\section{Fehler 1. Art}

R.-D. Hilgers ${ }^{1}$, N. Heussen ${ }^{1}$ und S. Stanzel ${ }^{2}$

${ }^{1}$ Institut für Medizinische Statistik, Universitätsklinikum der RWTH Aachen, Aachen, Deutschland

${ }^{2}$ DKFZ Heidelberg, Heidelberg, Deutschland

Synonym(e) $\alpha$-Fehler

\section{Englischer Begriff type I error}

Definition Ein Fehler 1. Art liegt vor, wenn auf der Basis eines statistischen Tests ( $\triangleright$ Test, statistischer) eine Entscheidung für die Annahme der $>$ Alternativhypothese getroffen wird, obwohl die $>$ Nullhypothese gültig ist.
Beschreibung Der Fehler 1. Art stellt eine mögliche Fehlentscheidung eines statistischen Tests dar. Entscheidet man sich auf der Basis eines statistischen Tests fälschlicherweise für die Ablehnung der Nullhypothese, begeht man einen Fehler 1. Art. Die Wahrscheinlichkeit für einen Fehler 1. Art wird üblicherweise als $>$ Irrtumswahrscheinlichkeit $\alpha$ bezeichnet. Bei der Anwendung statistischer Tests wird der Wert von $\alpha$ a priori festgelegt.

Analog spricht man im diagnostischen Test $(\triangleright$ Test, diagnostischer) von einem falsch-positiven Testergebnis ( $\triangleright$ Testergebnis, falsch-positives).

\section{Literatur}

Hilgers R-D, Bauer P, Scheiber V (2002) Einführung in die Medizinische Statistik. Springer, Berlin/Heidelberg/New York 